\title{
A technique for the independent-axis calibration of robot manipulators with experimental verification
}

\author{
Ibrahim A. Sultan \\ School of Engineering \\ University of Ballarat \\ PO BOX 663 \\ Ballarat 3353 VIC \\ Australia

\section{John G. Wager} \\ Department of Mechanical and Materials Engineering \\ The University of Western Australia \\ Nedlands WA 6907 \\ Australia
}

\begin{abstract}
:
Accurate use of robots in an off-line programming mode is only possible through a proper calibration procedure. In this procedure, the end-effector is made to move along a set of known spatial poses where the positional errors are to be measured and employed in mathematical models. The models are subsequently solved for the manipulator dimensions (geometric parameters) using suitable regression techniques.
\end{abstract}

Calibration is usually performed using either aggregate or independent-axis models. While the aggregate models result in all the system parameters worked out simultaneously, the independent-axis models are meant to work out the geometric particulars of each join-axis individually. In the present work, the independent-axis 
technique is used for the analysis with new mathematical models proposed to overcome the drawbacks of the existing methods.

Moreover, the techniques employed here result in the prediction of transmission error functions and the modelling of the joint motion dependencies. This is a new concept in the field of robot calibration. Finally, the models proposed have been used to calibrate an ASEA IRB/L6 robot and the results are reported at the end of the paper.

\section{Introduction and Literature Review}

Robots exhibit considerable positioning errors when they carry out tasks programmed off-line, and therefore this useful programming technique is rarely utilised in industrial applications. Positioning errors may reveal that the true dimensions of the manipulator are different from those used in the mathematical models. These dimensions, which are usually referred to as geometric parameters, undergo changes during manufacture and assembly.

In the majority of the published literature, the actual values of the geometric parameters of a manipulator are identified through the use of an aggregate differential model. The positioning errors measured at the end-effector are related to the infinitesimal changes of all parameters involved in the kinematic model by the use of a Jacobian matrix. This matrix requires a considerable amount of time to calculate at each iteration. Hayati et al (1988) and Zhuang et al (1990) discuss techniques they have used to work out this matrix. 
Since the number of model parameters exceeds the number of the spatial position parameters of the end-effector (which is $\leq 6$ ), a sufficient amount of data has to be collected by measuring the positioning errors of the end-effector at many locations. The methods of Least-Squares and Kalman filters are the two regression techniques usually employed for the estimation analysis as explained by Mooring et al (1991). LeastSquares is an estimation technique by which a batch of pose-error data acquired at many locations is fed to a computer program. The programs usually implement linear algorithms since no significant improvement would be achieved by using the non-linear Least Squares methods as discovered by Driels and Pathre (1991), who also discovered that the measurement strategy has a considerable impact on the outcome of the calibration work. In other words, the calibration results are influenced by the number of joints that are commanded to move and the amount of displacement allowed. This is particularly relevant to calibration techniques which involve aggregate models.

Whitney et al (1986) and Trevelyan (1996), replace the regression technique of LeastSquares by the Kalman filter method which offers the possibility of online parameters estimation. Bay (1993), therefore, proposes a theoretical technique by virtue of which the robot can be made to move from one position to another while the errors are being registered and used to calculate the actual values of the model parameters on the fly. Naturally these values will progressively improve as the calibration proceeds.

Aggregate differential models are sensitive to robot configurations at which the measurements are acquired irrespective of the method used to obtain the solution. The 
optimum configuration is defined by the set of joint-displacements at which the impact of measurement noise is minimised and the effects of parameter-errors are clearly reflected on the measured pose of the end-effector. The problem of selecting best configurations for measurements was tackled in works by Menq et al (1989), Khalil et al (1993) and Zhuang et al (1994) who pointed out that an optimum solution to this problem requires tremendous computing capabilities which are not yet available. Either the singular values of the Jacobian or its condition number are usually employed to select measurement configurations as detailed by Mooring et al (1991), Driels and Pathre (1990), Khalil et al (1991) to mention a few.

In most of the published literature, the spatial orientation of the end-effector is excluded from the analysis because it is not always possible, or economical, to measure spatial angles. This would adversely affect the outcome of any calibration process performed by aggregate models even though it is generally believed that such an effect is minimal.

The independent-axis approach is also implemented for robot calibration where the robot is commanded to move one joint at a time and the location of an auxiliary point is measured as it negotiates a path about or along the joint-axis. The data collected for the path points is then used to calculate the spatial location of the corresponding joint-axis. The method does not require the use of sophisticated equipment to measure six position parameters at the end-effector neither does it involve the lengthy computations of a large Jacobian matrix. Also the values of geometric parameters obtained by this method would be much closer to the true quantities than those values obtained by the aggregate model 
techniques. Therefore, the method is more suitable for applications where a diagnostic report on the error sources is to be produced such that these sources may be isolated and corrected. Stone and Sanderson (1987), Broderick and Cipra (1988), Kim et al (1990) and Stanton and Parker (1992) successfully implemented this approach

It has been argued that the method of independent-axis does not produce information on joint transmission errors. This was noted by Hollerbach and Wampler (1996) who refer to the independent axis method as the "circle point analysis" (CPA). However, the assertion in this paper is that the data collected for the analysis of independent-axis can conveniently be used to calculate profiles for transmission errors if appropriate mathematical models are implemented as will be shown below.

\section{Joint-Axis Identification}

The core idea of the independent-axis technique is to move the robot about one joint-axis at a time and use the resulting position vectors of an observed point to work out the spatial particulars of the axis. The path of the observed point can be either a circular arc or a straight line depending on whether the joint investigated was of a rotary or a sliding type respectively. Any such technique should not involve the joint-displacements in the analysis since it is most likely that the actual values of these displacements would be different from the commanded quantities as a result of the joint transmission errors and transducer offsets. Therefore if these quantities are used on face value in the mathematical model they are likely to produce unreliable outcome. The independent-axis techniques proposed by Broderick and Cipra (1988) and Kim et al (1990) involve these 
displacements in the analysis. The models proposed in the present paper, however, are devoid of any reference to joint displacements and as such produce more accurate results.

Kinematic models should be examined carefully before employed for robot calibration in order to reduce the occurrence of mathematical complications and misleading outputs. For example, the DH-representation, which was proposed, by Denavit and Hartenberg (1956) produces a mathematical singularity rendering it unsuitable for robot calibration, particularly if two successive joints on the robotic structure were designed to have their axes parallel in space. Therefore a kinematic notation published by Sultan and Wager (1999) was employed in the present work for the analysis. The notation, which is referred to by as the $\phi$-model, is described in the appendix.

\subsection{Calibration of an Axis of Rotation:}

Figure (1) depicts the axis, $\mathrm{Z}_{i}$, of a rotary joint number $(i)$ on a manipulator structure. The base frame, $\mathrm{X}_{0} \mathrm{Y}_{0} \mathrm{Z}_{0}$, is also shown in the figure, and so is the $\phi_{0}$-frame, as established in the order presented in the appendix. The stationary base frame may be selected to coincide with a frame attached to the measuring instrument. Point $\mathrm{p}^{i 0}$, in the figure refers to the initial location of an auxiliary point whose spatial position is affected by rotational displacements performed about the axis $Z_{i}$. In the current context the position vector, $\mathbf{p}_{0}^{i 0}$, of point $\mathrm{p}^{i 0}$ is accurately measured with respect to the base frame. 
The position vector, $\mathbf{p}_{i}^{i 0}$, of the same point with respect to the $i$-frame can be expressed as follows;

$\left[\begin{array}{c}\mathbf{p}_{i}^{i 0} \\ 1\end{array}\right]={ }_{i} \mathbf{T}^{0}\left[\begin{array}{c}\mathbf{p}_{0}^{i 0} \\ 1\end{array}\right]$

where ${ }_{i} \mathbf{T}^{0}$ is the matrix which performs the transformation from the base frame to the $i$-frame. This matrix may be calculated as follows;

${ }_{i} \mathbf{T}^{0}=\left({ }_{\phi_{0}} \mathbf{T}^{i}\right)^{-1}\left({ }_{0} \mathbf{T}^{\phi_{0}}\right)^{-1}$

where both of ${ }_{\phi_{0}} \mathbf{T}^{i}$ and ${ }_{0} \mathbf{T}^{\phi_{0}}$ are constructed as detailed in the Appendix. These matrices encompass the geometric parameters which relate the $i$-frame to the base frame according to the $\phi$-model conventions.

It may be noted here that the matrix ${ }_{i} \mathbf{T}^{0}$ does not include any reference to the displacement performed about the axis of rotation. The only displacement referred to in this matrix is the one performed about the Z-axis of the base frame which may be, conveniently, set equal to zero. This property will result in the joint-displacement being eliminated from the analysis in order to achieve a desirable outcome.

The displaced location, $\mathrm{p}^{i j}$, of point $\mathrm{p}^{i 0}$ may be measured with respect to the base frame using the position vector $\mathbf{p}_{0}^{i j}$. The position vector, $\mathbf{p}_{i}^{i j}$, of the displaced point with respect to the $i$-frame may be worked out in a concept similar to that presented in equation (1). 
After sufficient data have been collected by moving the observed point $n$ times about the axis $Z_{i}$; two cost functions may be minimised. The first function, $f_{1}$, represents the collective errors measured along the axis $\mathrm{Z}_{i}$. This function may be calculated as follows;

$f_{1}=\sum_{j=1}^{n-1}\left(p_{i z}^{i j}-p_{i z}^{i 0}\right)^{2}$

where $p_{i z}^{i 0}$ and $p_{i z}^{i j}$ are the $\mathrm{Z}$-components of $\mathbf{p}_{i}^{i 0}$ and $\mathbf{p}_{i}^{i j}$ respectively.

The second cost function, $f_{2}$, encompasses the collective radial errors measured about the axis $\mathrm{Z}_{i}$. This function is worked out as follows;

$$
f_{2}=\sum_{j=1}^{n-1}\left\{\left(p_{i x}^{i j}-p_{i x}^{i 0}\right)\left(p_{i x}^{i j}+p_{i x}^{i 0}\right)+\left(p_{i y}^{i j}-p_{i y}^{i 0}\right)\left(p_{i y}^{i j}+p_{i y}^{i 0}\right)\right\}^{2}
$$

where $p_{i x}^{i 0}$ and $p_{i x}^{i j}$ are the $\mathrm{X}$-components of the position vectors $\mathbf{p}_{i}^{i 0}$ and $\mathbf{p}_{i}^{i j}$ respectively and $p_{i y}^{i 0}$ and $p_{i y}^{i j}$ are their corresponding $\mathrm{Y}$-components.

The final solvable form of the proposed model is therefore given as follows;

$$
\left[\begin{array}{c}
\delta a \\
\delta b \\
\delta \alpha \\
\delta \beta
\end{array}\right]=\left(\mathbf{J}^{T} \mathbf{J}\right)^{-1} \mathbf{J}^{T} \mathbf{e}
$$

where $\mathbf{J}$ is the system $2(n-1) \times 4$ Jacobian matrix and $\mathbf{e}$ is the aggregate error vector. In the equation, $\delta a, \delta b, \delta \alpha$ and $\delta \beta$ refer to differential changes in their corresponding geometric parameters. 
A standard iterative technique may be implemented to calculate the actual values of the model parameters. These values are then used to locate the joint-axis in space with respect to the base frame.

After the spatial particulars of the calibrated axis have been obtained, the observed points may be projected onto a plane normal to the axis where the actual rotational displacements can be easily calculated. These displacements may then be related to their corresponding commanded values such that the actual kinematic models of the motiontransmission linkages and gears may be calculated. The projected two-element vector, $\mathbf{v}_{i}^{i j}$, which corresponds to point $\mathrm{p}^{i j}$ may be expressed as follows;

$\mathbf{v}_{i}^{i j}=p_{i x}^{i j} \mathbf{x}_{i}+p_{i y}^{i j} \mathbf{y}_{i}$

where $p_{i x}^{i j}$ and $p_{i y}^{i j}$ are defined above and $\mathbf{x}_{i}$ and $\mathbf{y}_{i}$ are unit vectors along the $\mathrm{X}_{i}$-axis and $\mathrm{Y}_{i}$-axis respectively.

The angular displacement $\theta_{i}^{j}$ which causes the observed point to travel from the location $\mathrm{p}^{i(j-1)}$, with a corresponding projected vector $\mathbf{v}_{i}^{i(j-1)}$, to the current location may be calculated as follows;

$\theta_{i}^{j}=\operatorname{signof}\left(\mathbf{v}_{i}^{i j} \times \mathbf{v}_{i}^{i(j-1)} \circ \mathbf{z}_{i}\right) \tan ^{-1}\left\{\frac{\left|\mathbf{v}_{i}^{i j} \times \mathbf{v}_{i}^{i(j-1)}\right|}{\mathbf{v}_{i}^{i j} \circ \mathbf{v}_{i}^{i(j-1)}}\right\}$

where $\mathbf{z}_{i}$ is a unit vector in the direction of $Z_{i}$. 
The observed displacement $\theta_{i}^{j}$ can be related mathematically to the commanded displacement $\theta_{i c}^{j}$ by an appropriate function, $f_{i}\left(\theta_{i}^{j}\right)$, whose constants may be calculated using a suitable least-squares regression technique. This can be achieved by minimising the error function, $e_{i}$, which is given as follows;

$e_{i}=\sum_{j=1}^{n-1}\left(\theta_{i c}^{j}-f_{i}\left(\theta_{i}^{j}\right)\right)^{2}$

where $n$ is the number of points observed.

The mathematical form assigned to the error function may be selected to comply with the kinematic nature of the motion-transmission linkages in question or to satisfy any desired statistical characteristics.

\subsection{Calibration of an Axis of Sliding:}

Figure (2) depicts the axis, $\mathrm{Z}_{i}$, of a sliding joint number (i) on a manipulator structure. The stationary base frame, $\mathrm{X}_{0} \mathrm{Y}_{0} \mathrm{Z}_{0}$, is also shown in the figure as is the $\phi_{0}$-frame which is established in the order presented above. The stationary base frame may be established at the measuring instrument. Point $\mathrm{p}^{i 0}$ in the figure refers to the initial location of an auxiliary point whose spatial position is affected by linear displacements performed along the axis $Z_{i}$. As mentioned in the previous section, the position vector, $\mathbf{p}_{0}^{i 0}$, of point $\mathrm{p}^{i 0}$ is accurately measured with respect to the base frame. The position vector, $\mathbf{p}_{i}^{i 0}$, of the same point with respect to the $i$-frame can be calculated using equation (1). 
It may be noted that, in this case, the auxiliary point will always move along a line parallel to the direction of the $\mathrm{Z}_{i}$-axis and therefore only the direction of this axis is relevant to the analysis. As such, the following mathematical identities may be proposed for any sliding joint-axis on an open-chain manipulator structure;

$a_{i}=0$

and

$b_{i}=0$

The displaced location, $\mathrm{p}^{i j}$, of point $\mathrm{p}^{i 0}$ may be measured with respect to the base frame using the position vector $\mathbf{p}_{0}^{i j}$. The position vector, $\mathbf{p}_{i}^{i j}$, of the displaced point with respect to the $i$-frame may be worked out in a concept similar to that present in equation (1).

After sufficient data have been collected by moving the observed point $n$ times along the axis $\mathrm{Z}_{i}$, the two cost functions , $f_{1 s}$ and $f_{2 s}$, which represent the collective errors measured in the $\mathrm{X}$-direction and $\mathrm{Y}$-direction respectively can be minimised. These functions can be expressed as follows;

$$
f_{1 s}=\sum_{j=1}^{n-1}\left(p_{i x}^{i j}-p_{i x}^{i 0}\right)^{2}
$$

and

$$
f_{2 s}=\sum_{j=1}^{n-1}\left(p_{i y}^{i j}-p_{i y}^{i 0}\right)^{2}
$$

where the variables hold the same definitions given in the previous section. 
The final form of the proposed model is therefore given as follows;

$\left[\begin{array}{l}\delta \alpha \\ \delta \beta\end{array}\right]=\left(\mathbf{J}_{s}^{T} \mathbf{J}_{s}\right)^{-1} \mathbf{J}_{s}^{T} \mathbf{e}_{s}$

where $\mathbf{J}_{s}$ is the system $2(n-1) \times 2$ Jacobian matrix and $\mathbf{e}_{s}$ is the aggregate error vector while $\delta \alpha$ and $\delta \beta$ refer to differential changes in their corresponding geometric parameters.

A standard iterative technique may be implemented to calculate the actual values of the two model parameters. These values are then used to determine the actual orientation of the joint-axis in space with respect to the Cartesian base frame.

To produce a mathematical model to describe the actual kinematic behaviour of the motion-transmission linkages, the observed value of linear displacement, $S_{i}^{j}$, may be calculated as follows;

$S_{i}^{j}=p_{i z}^{i j}-p_{i z}^{i(j-1)}$

where $p_{i z}^{i j}$ and $p_{i z}^{i(j-1)}$ are the $\mathrm{Z}_{i}$-components of both of $\mathbf{p}_{i}^{i j}$ and $\mathbf{p}_{i}^{i(j-1)}$ respectively.

The observed displacement can then be related mathematically to the commanded displacement $S_{i c}^{j}$ by an appropriate function, $f_{i s}\left(S_{i}^{j}\right)$, whose coefficients may be calculated using a suitable Least-Squares regression technique. This can be achieved by minimising the error function, $e_{i s}$, which is given as follows;

$e_{i s}=\sum_{j=1}^{n-1}\left(S_{i c}^{j}-f_{i}\left(S_{i}^{j}\right)\right)^{2}$ 
where $n$ is the number of points observed.

\section{Calibration of Manipulators with Kinematic Dependencies}

Commercial robots are usually equipped with certain level of rigidity to render them suitable for use within industrial environments. To achieve the required level of rigidity mechanical linkages are used instead of simple gear-trains to transmit power to the manipulator joints. This usually results in motions performed at some joints being transmitted to other joints on the manipulator structure. Naturally, the resulting kinematic dependencies are accounted for in the nominal mathematical models stored in the controller so that the correct displacement inputs are issued to the actuator servos.

The ASEA IRB/L6 robot, which is employed in the present work for experimentation, possesses a set of joints whose motions are characterised with kinematic dependencies. The manner in which the controller handles the resulting kinematic dependencies is detailed in figure (3). In this figure, a box designates a functional transformation of the signal shown on the left-hand side of the box to the signal resulting on the opposite side and the summing point implies addition or subtraction as indicated. As shown in the figure, the required pose command is used by the controller in an inverse-position procedure to calculate the corresponding set of joint-displacements, $\boldsymbol{\theta}_{\mathrm{c}}$, which are then reduced by the current readings of displacement-transducers, $\theta_{\text {off }}$. This reduction, which is represented in the figure by the left-hand side summing points, may take place at any stage depending on where the transducers are located on the structure. Further subtractions take place to account for the mechanical dependencies between the different 
joints. The resulting quantities are used in the inverse kinematic models which correspond to the mechanisms driven by the various actuators. The inverse models are represented by the box at the left end of figure (3). The final outcome of this analysis encompasses the input commands as issued to the actuator servos.

\subsection{Motion Decoupling:}

As shown in figure (3), the kinematic dependencies often carry the following two characteristics which simplify the mathematical modelling of their kinematic behaviour;

- They usually occur between two successive joints where motion is only imparted in an upwards-counting direction starting from the base. In other words the motions occurring about joint-axis number $(i)$ will be transmitted to joint-axis number $(i+1)$ and not the opposite. The motion imparted to a joint by virtue of its actuator is referred to here as the active motion. Passive motions occur about joint-axis number $(i+1)$ by the virtue of actuator number $(i)$. The net motion occurring about a joint-axis is the algebraic sum of both the active and passive motions taking place about that axis. This is represented in the figure by the right-hand side summing points.

- Joint number (i) may impart only its active motion to joint number $(i+1)$. Consequently, passive motions do not travel more than one step upwards on the manipulator structure, thus workable simplified kinematic models may be obtained.

A careful investigation into the aspects of these characteristics reveals that joint-axes may be calibrated in a descending order starting from the one attached to the end- 
effector. Indeed these two characteristics present an opportunity for the independent-axis procedures, such as the one used in the current work, to describe the actual kinematic behaviour of not only the motion-transmission mechanisms but also the motion dependencies among the successive joints on the manipulator structure in a decoupled fashion. This may be achieved as follows,

1. The manipulator is made to move joint number $(i+1)$ such that the spatial location of the joint-axis and its associated mechanical transmission errors may be calculated by observing the locations of a suitable point on the arm structure.

2. The actuator of joint number $(i)$ is then made to move while all other motors (including $i+1$ ) are held still. This can be done by issuing positioning-commands, that have been calculated by the nominal dependency models, to joint $(i+1)$. The commands would include displacements equal to the nominal passive displacements which the controller expects the motions of joint (i) to reflect on the state of joint number $(i+1)$. Therefore the controller will not issue displacement commands to the actuator of joint number $(i+1)$ and hence the motion measured about the axis of this joint may be attributed solely to the motion of joint number (i).

While motions are being performed about joint-axis number (i), two points may be observed in order to calibrate the spatial pose of this axis and the actual kinematic dependency models as detailed in the next section. 


\subsection{Modelling of Kinematic Dependencies:}

Reference is made in this section to figure (4) which indicates that the spatial position of the axis $Z_{i+1}^{j}$ is affected by motions performed about the stationary axis $Z_{i}$. It is assumed here that the initial spatial location of $Z_{i+1}$ and its actual motion-transmission model have already been calibrated. To measure the spatial particulars of $Z_{i}$, successive rotations about it may be performed where at each step, $j$, the location of a point, $\mathrm{p}^{i j}$, is observed for use in the model described in equations (1) through (8). During this experiment, positioning commands can be issued to the robot controller as explained above and the passive motion, $\theta_{i(i+1)}^{j}$, may be represented by a suitable mathematical function using the procedure described below.

Figure (4) depicts point $\mathrm{p}^{(i+1) j}$ which lies on the $(i+1)$-frame. The point is therefore affected by displacements performed about both $Z_{i}$ and $Z_{i+1}^{j}$. At step number $(j)$, the point $\mathrm{p}^{(i+1) j}$ is observed and its spatial location with respect to the base frame, $\mathbf{p}_{0}^{(i+1) j}$, is therefore measured. The location of this point with respect to the $Z_{i+1}^{j}$-frame, $\mathbf{p}_{i+1}^{(i+1) j}$, may be expressed as follows;

$\left[\begin{array}{c}\mathbf{p}_{i+1}^{(i+1) j} \\ 1\end{array}\right]=\left({ }_{\phi_{i}} \mathbf{T}^{i+1}\right)^{-1}\left({ }_{i} \mathbf{T}^{\phi_{i}}\right)^{-1}\left({ }_{\phi_{0}} \mathbf{T}^{i}\right)^{-1}\left({ }_{0} \mathbf{T}^{\phi_{0}}\right)^{-1}\left[\begin{array}{c}\mathbf{p}_{0}^{(i+1) j} \\ 1\end{array}\right]$

where all the entries of the T-matrices are fully defined. 
The projection of $\mathbf{p}_{i+1}^{(i+1) j}$ onto a plane perpendicular to $Z_{i+1}^{j}$ may be calculated in a manner similar to that presented in equation (6) and compared with the projection of $\mathbf{p}_{i+1}^{(i+1)(j-1)}$ in order to work out the passive angular displacement, $\theta_{i(i+1)}^{j}$. This displacement may now be related to the active displacement, $\theta_{i}^{j}$, which occurs about $\mathrm{Z}_{i}$, using an appropriate function, $f_{i(i+1)}\left(\theta_{i}^{j}\right)$. The coefficients of this function may be calculated using a suitable least-squares technique where the following function, $e_{i(i+1)}$, may be minimised;

$e_{i(i+1)}=\sum_{j=1}^{n-1}\left(\theta_{i(i+1)}^{j}-f_{i(i+1)}\left(\theta_{i}^{j}\right)\right)^{2}$

where $n$ in the above equation designates the number of observations performed.

The strategy of the error-compensation software proposed for the robot under investigation is shown in figure (5). The figure contains reference to the mathematical functions obtained, from the calibration procedure, to describe the kinematic behaviour of various linkages on the manipulator structure. As shown in the figure, the required joint-displacements will undergo transformations through these functions before the initial transducer offsets are added. The resulting displacements will then be used in a nominal direct kinematic analysis, the outcome of which will be sent to the robot controller. 
In the next section, the numerical results of the calibration work are presented together with the different functions which describe the kinematic behaviour of the motiontransmission linkages.

\section{Experimental Results}

The mathematical models and concepts presented in the previous sections was used to calibrate the six-axis ASEA IRB6/L industrial manipulator whose schematic diagram is shown in figure (6). The positioning error of the particular robot tested was as high as 47.0mm which reveals a serious level of inaccuracy.

Theodolites were selected as data-acquisition media for the calibration work described in this paper. Theodolites represent a class of well-established and accurate technology most suitable for 3D point measurement, which is of particular importance in independent axis calibration techniques.

Before the phase of data acquisition started, two theodolites were secured to the laboratory floor along a line approximately 3.0 meters away from the manipulator under calibration. A general view of layout of the experimental set up is shown in figure (7), where the theodolites appear at the right side of the photograph and the robot calibrated is situated at the left-most side.

The measurement base coordinate system, with respect to which the spatial particulars of the axes were to be calibrated, was set up at the LHS theodolite and the procedure 
presented above was used to move the robot about one joint-axis at a time, while corresponding target points were being observed. The data collected were then used in the models described above to work out the actual spatial particulars of the joint-axes and the kinematic behaviour of the motion-transmission linkages and gears. The particulars of the relevant calibration data and the order in which these data were collected are briefly described in table (1).

The selection of the displacement ranges displayed in this table was controlled by the observability of the relevant target points with respect to theodolites. The calibration results are given as follows,

- Joint-axis number (6) was calibrated and its spatial particulars are found to be;

$$
\begin{aligned}
& \mathbf{p}_{0}^{6}=-3.1565 \mathbf{x}_{0}+5.3466 \mathbf{y}_{0}-0.52353 \mathbf{z}_{0} \\
& \mathbf{z}_{0}^{6}=-0.006872 \mathbf{x}_{0}+0.002904 \mathbf{y}_{0}-0.999972 \mathbf{z}_{0}
\end{aligned}
$$

where $\mathbf{p}_{0}^{6}$ is the position vector of a point on the joint-axis and $\mathbf{z}_{0}^{6}$ is the orientation of the axis. $\mathbf{x}_{0}, \mathbf{y}_{0}$ and $\mathbf{z}_{0}$ are unit vectors along the corresponding directions of the base frame. The various elements of the position vector above are given in meters which will also apply to other position vectors given below.

The function, $f\left(\theta_{6}^{\text {act }}\right)$, which represents the kinematic behaviour of the motiontransmission gears was evaluated as;

$$
\theta_{6}^{c}=\theta_{6}^{\text {act }}+0.017334 \sin \left(\theta_{6}^{\text {act }}\right)+0.002677 \cos \left(\theta_{6}^{\text {act }}\right)
$$


where $\theta_{6}^{c}$ is the value of the joint displacement command issued to the controller and $\theta_{6}^{a c t}$ is the actual observed displacement. Both angles are given in radians as are all other angles described below.

\section{The Wrist-Joints:}

A schematic diagram of the wrist-joints (No's. 4 and 5) is shown in figure (8). As indicated in the figure, the motions are transmitted from the joint-actuators, which are located near the robot base, to the joint-shafts via over-constraint linkages. An over-constraint mechanism usually has a calculated mobility less than its actual number of degrees-of-freedom indicating that its components have to be manufactured up to a high degree of accuracy or it would otherwise seize. Figure (8) also indicates that rotations performed by the bevel gear, G, of joint number (5) will not affect the state of the fourth joint. However, any rotations performed by the later joint will cause the small bevel gear, g, to roll over $G$ and thus creating passive rotations, $\theta_{45}^{\text {act }}$, about the axis of the fifth joint.

- Joint-axis number (5) was calibrated, separately prior to the fourth joint-axis, and its spatial particulars are,

$$
\begin{aligned}
& \mathbf{p}_{0}^{5}=-3.14308 \mathbf{x}_{0}+4.44598 \mathbf{y}_{0}-0.16303 \mathbf{z}_{0} \\
& \mathbf{z}_{0}^{5}=-0.014096 \mathbf{x}_{0}+0.99990 \mathbf{y}_{0}+0.000597 \mathbf{z}_{0}
\end{aligned}
$$

- Joint-axis number (4) was then calibrated and its spatial particulars are,

$$
\begin{aligned}
& \mathbf{p}_{0}^{4}=-3.1367 \mathbf{x}_{0}+5.1116 \mathbf{y}_{0}+0.16354 \mathbf{z}_{0} \\
& \mathbf{z}_{0}^{4}=0.999917 \mathbf{x}_{0}+0.012724 \mathbf{y}_{0}+0.002198 \mathbf{z}_{0}
\end{aligned}
$$


Due to the over-constrained nature of the mechanism involved, the function, $f\left(\theta_{4}^{a c t}\right)$, which describes the kinematic behaviour of the active motion of this joint may be approximated by;

$\theta_{4}^{c}=\theta_{4}^{a c t}$

During the calibration of the fourth joint-axis the actuator of joint-axis number (5) was held still (by issuing the appropriate positioning commands to the controller) in order that the actual passive motion, $\theta_{45}^{\text {act }}$, transmitted from joint (4) to joint number (5) may be modelled in a function, $f_{45}\left(\theta_{4}^{\text {act }}\right)$. This function takes a form suitable to accommodate errors in the gear train which drives the fifth joint and is given as follows,

$\theta_{45}^{\text {act }}=N \theta_{4}^{\text {act }}-0.040562 \sin \left(N \theta_{4}^{\text {act }}\right)+0.000018 \cos \left(N \theta_{4}^{\text {act }}\right)$

where the gear ratio, $N$, is equal to $32 / 19$ as per the maintenance manual supplied by the robot manufacturer.

The function, $f\left(\theta_{5}^{\text {act }}\right)$, which describes kinematic behaviour of the independent motion of joint number (5) is therefore approximated as follows;

$\theta_{5}^{c}=\theta_{5}^{G}$ 
where $\theta_{5}^{G}$ is the corresponding displacement performed at the bevel gear, G, which drives the fifth joint. $\theta_{5}^{G}$ may be calculated by using the following equation, $\theta_{5}^{G}=\frac{1}{N} \theta_{5}^{a c t}+0.041227 \sin \left(\frac{1}{N} \theta_{5}^{a c t}\right)-0.000013 \cos \left(\frac{1}{N} \theta_{5}^{a c t}\right)$

\section{The Arm-Joints:}

A schematic diagram of the arm-joints (No's 2 and 3) is shown on the left-hand side of figure (6). As indicated in the figure, the motions are transmitted from the joint-actuators, which are located near the robot base, to the joint-axes via mechanical linkages. Independent powering of joint number (3) will cause link number (1) to remain stationary such that the state of the second joint is not affected. However, the motions about the axis of joint number (2) will cause link number (2) to become stationary, thus creating passive motions, $\theta_{23}^{\text {act }}$, about the axis of the third joint.

- Joint-axis number (3) was calibrated, separately prior to the second joint-axis, and its spatial particulars were found to be;

$$
\begin{aligned}
& \mathbf{p}_{0}^{3}=-3.1367 \mathbf{x}_{0}+4.4388 \mathbf{y}_{0}+0.1638 \mathbf{z}_{0} \\
& \mathbf{z}_{0}^{3}=0.999983 \mathbf{x}_{0}+0.00537 \mathbf{y}_{0}+0.002136 \mathbf{z}_{0}
\end{aligned}
$$

The function, $f\left(\theta_{3}^{\text {act }}\right)$, which describes the kinematic behaviour of the active motion of this joint is calibrated to be;

$$
\theta_{3}^{c}=\theta_{3}^{\text {act }}+0.003462 \sin \left(\theta_{3}^{\text {act }}\right)-0.000867 \cos \left(\theta_{3}^{\text {act }}\right)
$$


Even though fractional forms were experimented with to describe this function, the only form which was proven to give most accurate results is the one displayed above.

The function, $f_{34}\left(\theta_{3}^{\text {act }}\right)$, which describes the transmission of passive motion from the third to the fourth joint is simply given as follows;

$$
\theta_{34}^{a c t}=\theta_{3}^{a c t}
$$

- Joint-axis number (2) was calibrated and its spatial particulars are found to be;

$$
\begin{aligned}
& \mathbf{p}_{0}^{2}=-3.1352 \mathbf{x}_{0}+4.4442 \mathbf{y}_{0}-0.5293 \mathbf{z}_{0} \\
& \mathbf{z}_{0}^{2}=0.999898 \mathbf{x}_{0}+0.013323 \mathbf{y}_{0}+0.0051096 \mathbf{z}_{0}
\end{aligned}
$$

The function, $f\left(\theta_{2}^{\text {act }}\right)$, which describes the kinematic behaviour of the active motion of this joint is given as follows;

$$
\theta_{2}^{c}=\theta_{2}^{\text {act }}+0.001094 \sin \left(\theta_{2}^{\text {act }}\right)+0.001317 \cos \left(\theta_{2}^{\text {act }}\right)
$$

During the calibration of joint 2, the actuator which drives the third joint was held still such that the passive motion transmitted from joint (2) to joint (3) is described by the function, $f_{23}\left(\theta_{2}^{a c t}\right)$. The computed form of this function is as follows;

$$
\theta_{23}^{\text {act }}=\tan ^{-1}\left(\frac{-0.998132 \sin \left(\theta_{2}^{\text {act }}\right)+0.213253 \cos \left(\theta_{2}^{\text {act }}\right)-0.212863}{\cos \left(\theta_{2}^{\text {act }}\right)+0.096654 \sin \left(\theta_{2}^{\text {act }}\right)-0.0006941}\right)
$$


The fractional form of this function was selected to reflect the geometric aspects of the four-bar chain employed to transmit motion to the two joints as shown in figure (6).

- Joint-axis number (1) was calibrated and its spatial particulars are found to be;

$$
\begin{aligned}
& \mathbf{p}_{0}^{1}=-3.1423 \mathbf{x}_{0}+4.4394 \mathbf{y}_{0}-0.526123 \mathbf{z}_{0} \\
& \mathbf{z}_{0}^{1}=0.001445 \mathbf{x}_{0}+0.002876 \mathbf{y}_{0}+0.999995 \mathbf{z}_{0}
\end{aligned}
$$

The function, $f\left(\theta_{1}^{a c t}\right)$, which describes the kinematic behaviour of the active motion of this joint was calibrated to be;

$$
\theta_{1}^{c}=\theta_{1}^{a c t}+0.000771 \sin \left(\theta_{1}^{a c t}\right)-0.000007 \cos \left(\theta_{1}^{a c t}\right)
$$

A robot base coordinate system, $\mathrm{X}_{1} \mathrm{Y}_{1} \mathrm{Z}_{1}$, has been established with its $\mathrm{Z}$-axis directed along the calibrated axis of the first joint. The common normal directed from the axis of the first joint to the second joint-axis coincides with the Y-axis of the base system. The point at which this normal intersects the first joint-axis is the origin of the base coordinate system. The X-axis of the base coordinate system is then assigned to complete a right-handed Cartesian coordinate system.

Once the base coordinate system was assigned, $\phi$-frames were established around the different joint-axes using a computer program employing the convention described in the 
Appendix. The $\phi_{i}$-angles of the frames are equal for both the actual and nominal manipulators. These values are given as follows;

$\phi_{1}=\pi / 2, \phi_{2}=0.0, \phi_{3}=0.0, \phi_{4}=0.0$ and $\phi_{5}=0.0$.

The geometric parameters for both of the nominal and actual manipulators are given in table (2). A diagnostic report outlining the features which may have caused positioning errors to occur may then be compiled by studying the values of parameters in this table. Some of these features may be listed as follows;

- The values of parameters relevant to frames 1 and 2 reveal that the centre of the bearing of the second joint is about $2.3 \mathrm{~mm}$ away from its designed location along the axis of the first joint.

- The line connecting the second joint-axis to the third joint axis is about $0.37 \mathrm{deg}$ out of parallelism with the first-joint axis and the distance between the second and third axes is $3.3 \mathrm{~mm}$ wider than designed; however this distance is equal to the designed value when projected on the first axis.

- It may also be concluded from the values in table (2) that the distance between the two driving disks which lie along the fourth joint-axis is $2.9 \mathrm{~mm}$ wider than the designed value. However; the centres of the two disks are carefully aligned so that the overconstrained mechanism which drives the end-effector would not jam. This result 
would reveal that the geometric errors discovered here might have resulted from various levels of machining tolerances employed by the manufacturer.

\section{Testing the Performance}

To test the performance of the proposed models, a base coordinate-system was established about the axis of the first joint of the robot and related mathematically to a measuring frame constructed at the left-hand-side theodolite. Position commands were then issued to the robot controller after being processed by the compensation software as shown in figure (5) and the positions of three non-collinear points on the end-effector were observed by theodolites and compared with the required values such that both position and orientation errors of the end-effector could be calculated. The net radial error of the two-theodolite module used for data acquisition was evaluated at an earlier stage to be about $0.19 \mathrm{~mm}$.

The repeatable error of the ASEA IRB/L6 robot employed for the experimental work was tested by the commanding the robot to travel to a location within the work volume where the tool frame was observed using theodolites. The robot was then requested to execute the same command a number of times and the tool frame was observed and its particulars were compared to the first values measured. The mean values for the radial repeatable error and the orientation repeatable error are found to be $0.34 \mathrm{~mm}$ and 0.0033 radians respectively. 
The net radial error and orientation errors of both calibrated and uncalibrated robots are shown in figures (9) and (10) respectively. The calibration work carried out here resulted in the position error scaled down by a factor of about 33 times from $47.00 \mathrm{~mm}$ to $1.40 \mathrm{~mm}$ and the orientation error decreased from 0.05radians to 0.01radians. It may therefore be concluded that the calibration work performed has achieved the objective of improving the level of accuracy of the robot under study.

Other error sources (e.g. joint compliance) may have to be taken into consideration if higher degrees of accuracy are essential for the operation of the robot.

\section{Conclusion}

A new independent-axis calibration technique have been proposed and used to calibrate an ASEA IRB/L6 robot. The technique proposed does not suffer any of the drawbacks associated with existing methods and allow both the transmission errors and the joint kinematic dependencies to be included in the analysis. By virtue of the proposed technique, the robot was made to move about one axis at a time and the measured locations of an observed point were used to evaluate the location and orientation of that axis.

The calibration results reveal that the position error of the end-effector was significantly reduced thus affirming the effectiveness of the proposed technique. 


\section{Appendix: The $\phi$-model}

The kinematic aspects of the $\phi$-model notation are shown in figure (11). The model is established by introducing an intermediate Cartesian system between the joint-frames number (i) and (i+1). The Z-axis of the new frame, which is referred to as the $\phi_{i}$-frame, lies in a plane parallel to the $\mathrm{X}_{i} \mathrm{Y}_{i}$-plane and at a distance, $d_{i}$, equal to the linear jointdisplacement from it. In case of a rotary joint, $d_{i}$ may be set equal to zero. This Z-axis, which may be referred to as $\mathrm{Z}_{\phi_{i}}$, is initially set by the user at a constant angle, $\phi_{i}$, from the $\mathrm{X}_{i}$-axis. $\phi_{i}$, which is measured in a right-handed sense about $\mathrm{Z}_{i}$, is selected to ensure that $\mathrm{Z}_{\phi_{\mathrm{i}}}$ may not be parallel to $\mathrm{Z}_{i+1}$. The $\mathrm{X}_{\phi_{i}}$-axis of the $\phi_{i}$-frame is then established in a plane perpendicular to both $\mathrm{Z}_{\phi_{i}}$ and $\mathrm{Z}_{i}$. The $\phi_{i}$-frame is then used to establish a Cartesian system, $\mathrm{X}_{i+1} \mathrm{Y}_{i+1} \mathrm{Z}_{i+1}$, about the $\mathrm{Z}_{i+1}$-axis in a DH-fashion. The $\phi_{i}$-frame and the $(i+1)$ frame are on the same rigid link and perform the same displacement $\left(d_{i}\right.$ or $\left.\theta_{i}\right)$ along or about the $\mathrm{Z}_{i}$ respectively.

The transformation, $\mathbf{T}_{i}^{i+1}$, from the (i+1)-frame to the i-frame may now be expressed as follows,

${ }_{i} \mathbf{T}^{i+1}={ }_{i} \mathbf{T}^{\phi_{i}}{ }_{\phi_{i}} \mathbf{T}^{i+1}$ 
where ${ }_{i} \mathbf{T}^{\phi_{i}}$ and ${ }_{\phi_{i}} \mathbf{T}^{i+1}$ represent the transformation from the $\phi_{i}$-frame to the $i$-frame and from the (i+1)-frame to the $\phi_{i}$-frame respectively. These matrices may be expressed as follows,

${ }_{i} \mathbf{T}^{\phi_{i}}=\left[\begin{array}{cccc}-\sin \left(\phi_{i}+\theta_{i}\right) & 0 & \cos \left(\phi_{i}+\theta_{i}\right) & 0 \\ \cos \left(\phi_{i}+\theta_{i}\right) & 0 & \sin \left(\phi_{i}+\theta_{i}\right) & 0 \\ 0 & 1 & 0 & d_{i} \\ 0 & 0 & 0 & 1\end{array}\right]$

and

$$
\phi_{i} \mathbf{T}^{i+1}=\left[\begin{array}{cccc}
\cos \left(\beta_{i}\right) & -\sin \left(\beta_{i}\right) \cos \left(\alpha_{i}\right) & \sin \left(\beta_{i}\right) \sin \left(\alpha_{i}\right) & b_{i} \cos \left(\beta_{i}\right) \\
\sin \left(\beta_{i}\right) & \cos \left(\beta_{i}\right) \cos \left(\alpha_{i}\right) & -\cos \left(\beta_{i}\right) \sin \left(\alpha_{i}\right) & b_{i} \sin \left(\beta_{i}\right) \\
0 & \sin \left(\alpha_{i}\right) & \cos \left(\alpha_{i}\right) & a_{i} \\
0 & 0 & 0 & 1
\end{array}\right]
$$

where $a_{i}, b_{i}, \alpha_{i}$ and $\beta_{i}$ are the DH-parameters which relate the (i+1)-frame to the $\phi_{i}$-frame as shown in figure (11). As the above expression for $\mathbf{T}^{\phi_{i}}$ indicates, the angle between the $\mathrm{X}_{i}$ - and the $\mathrm{Z}_{\phi_{i}}$-axes is initially $\phi_{i}$. However with the onset of the rotational motion, this angle would vary by the value of the motor displacement, $\theta_{i}$. The expression also reveals that the $\phi_{i}$-frame may slide along the $\mathrm{Z}_{i}$-axis a distance $d_{i}$ if the joint was of the sliding type; in such a case $\theta_{i}$ may be set equal to zero.

To render the model complete such that arbitrarily-located frames (e.g. the tool frame, which may be predetermined by the requirements of some manufacturing set-up rather than assigned systematically according to the rules of the kinematic notation) can be described, a rotation, $\gamma_{i}$, and a translation, $h_{i}$, may be performed about and along the $\mathrm{Z}_{i+1^{-}}$ axis. The new (i+1)-frame can now be related to the $\phi_{i}$-frame by the following equation, 
$\phi_{i} \mathbf{T}^{i+1}=\operatorname{Trans}\left(0,0, a_{i}\right) \operatorname{Rot}\left(\mathbf{z}, \beta_{i}\right) \operatorname{Trans}\left(b_{i}, 0,0\right) \operatorname{Rot}\left(\mathbf{x}, \alpha_{i}\right) \operatorname{Rot}\left(\mathbf{z}, \gamma_{i}\right) \operatorname{Trans}\left(0,0, h_{i}\right)$

In a more expanded form equation (A3) can be re-expressed as follow;

$$
\begin{aligned}
& \phi_{\mathbf{T}^{i}} \mathbf{T}^{i+1}= \\
& {\left[\begin{array}{cccc}
\cos \left(\beta_{i}\right) & -\sin \left(\beta_{i}\right) \cos \left(\alpha_{i}\right) & \sin \left(\beta_{i}\right) \sin \left(\alpha_{i}\right) & \mathrm{b}_{i} \cos \left(\beta_{i}\right) \\
\sin \left(\beta_{i}\right) & \cos \left(\beta_{i}\right) \cos \left(\alpha_{i}\right) & -\cos \left(\beta_{i}\right) \sin \left(\alpha_{i}\right) & \mathrm{b}_{i} \sin \left(\beta_{i}\right) \\
0 & \sin \left(\alpha_{i}\right) & \cos \left(\alpha_{i}\right) & \mathrm{a}_{i} \\
0 & 0 & 0 & 1
\end{array}\right]\left[\begin{array}{cccc}
\cos \left(\gamma_{i}\right) & -\sin \left(\gamma_{i}\right) & 0 & 0 \\
\sin \left(\gamma_{i}\right) & \sin \left(\gamma_{i}\right) & 0 & 0 \\
0 & 0 & 1 & h_{i} \\
0 & 0 & 0 & 1
\end{array}\right]}
\end{aligned}
$$




\section{$\underline{\text { References: }}$}

1) Bay, J. S., 1993, Autonomous parameter identification by optimal learning control, IEEE J Control Systems, pp. 56-61.

2) Broderick, P. L. and Cipra, R. J., 1988, A method for determining and correcting robot position and orientation errors due to manufacturing, ASME J. Mechanisms, Transmission and Automation in Design, Vol. 110, pp. 3-10.

3) Denavit, J. and Hartenberg, R. S., 1956, A kinematic notation for low pair mechanisms based on matrices, ASME J. Appl. Mechanics, Vol. 22, pp. 215-221.

4) Driels, M. R. and Pathre, U. S., 1990, Significance of observation strategy on the design of robot calibration experiments, J Robotic Systems 7(2), pp. 197-223.

5) Driels, M. R. and Pathre, U. S., 1991, Vision-based automatic theodolite for robot calibration, IEEE Trans. on Robotics and Automation, Vol. 7, No. 3, pp. 351-360.

6) Hayati, S., Tso, K. and Roston, G., 1988, Robot geometry calibration, In Proceedings of IEEE Int. Conf. on Robotics and Automation, pp. 947-951.

7) Judd, R. P and Knasinski, A. B., 1991, A technique to calibrate industrial robots with experimental verification, IEEE Trans. on Robotics and Automation, Vol. 6, No. 1, pp. 20-30.

8) Kim, M. S., Yoo, H. S., Cho, S. W., Chang, H. S. and Spur, G., 1990, A new calibration technique, Annals of the CIRP, Vol. 39, pp. 421-424. 
9) Menq, C., Borm, J., Lai, J. Z., 1989, Identification and observability measure of a basic set of error parameters in robot calibration, ASME J. of Mechanisms, Transmissions and Automation in Design, Vol. 111, pp. 513-518.

10) Mooring, B. W., Roth, Z. S. and Driels, M. R., 1991, Fundamentals of Manipulator Calibration (New York: John Wiley \& Sons).

11) Stanton, D. and Parkar, G. A., 1992, Experimental kinematic calibration using a modified S-model, Proceedings of $2^{\text {nd }}$ Int. Conf. on Automation, Robotics and Computer Vision, Singapore, 15-18 Sept.

12) Stone, H. W. and Sanderson, A. C., 1987, A prototype arm signature identification system, Proceedings of IEEE Int. Conf. on Robotics and Automation, Raleigh, pp. $175-182$.

13) Stone, H. W., 1987, Kinematic Modelling, Identification and Control of Robotic Manipulators (Boston: Kluwer Academic Publishers).

14) Sultan, I. A. and Wager, J G., 1999, User-controlled kinematic modelling, Int. J. Advanced Robotics, Vol 12, No. 6.

15) Trevelyan, J. P., Legnani, J., Li, Z., Sultan, I. A., 1992, On robot calibration, Proceedings of AWToMM, Melbourne, Nov.

16) Trevelyan, J., 1996, Complete and parametrically continuous model for robot manipulator calibration, Technical Note, University of Western Australia. 
17) Van Brussel, H., 1990, Evaluation and testing of robots, Annals of the CIRP, Vol. 39/2, pp. 657-664.

18) Whitney, D. E., Lozinski, C. A. and Rourke, J. M., 1986, Industrial robot forward calibration method and results, ASME Journal of Dynamic Systems, Measurement and Control, Vol. 108, pp 1-8.

19) Zhuang, H., Roth, Z. S. and Hamano, F., 1990, A complete and parametrically continuous kinematic model for robot manipulators, Proceedings of IEEE Int. Conf. on Robotics and Automation, Cincinnati, pp. 92-97.

20) Zhuang, H., Wang, L. K. and Roth, Z. S., 1993, Error-model based robot calibration using a modified CPC model, J. Robotics and Computer Integrated Manufacturing, Vol. 10, pp. 287-299.

21) Zhuang, H., Wang, L. K. and Roth, Z. S., 1994, Optimal selection of measurement configurations for robot calibration using simulated annealing, Proceedings of IEEE Int. Conf. on Robotics and Automation, San Diego, 8-13 May, pp. 393-398. 


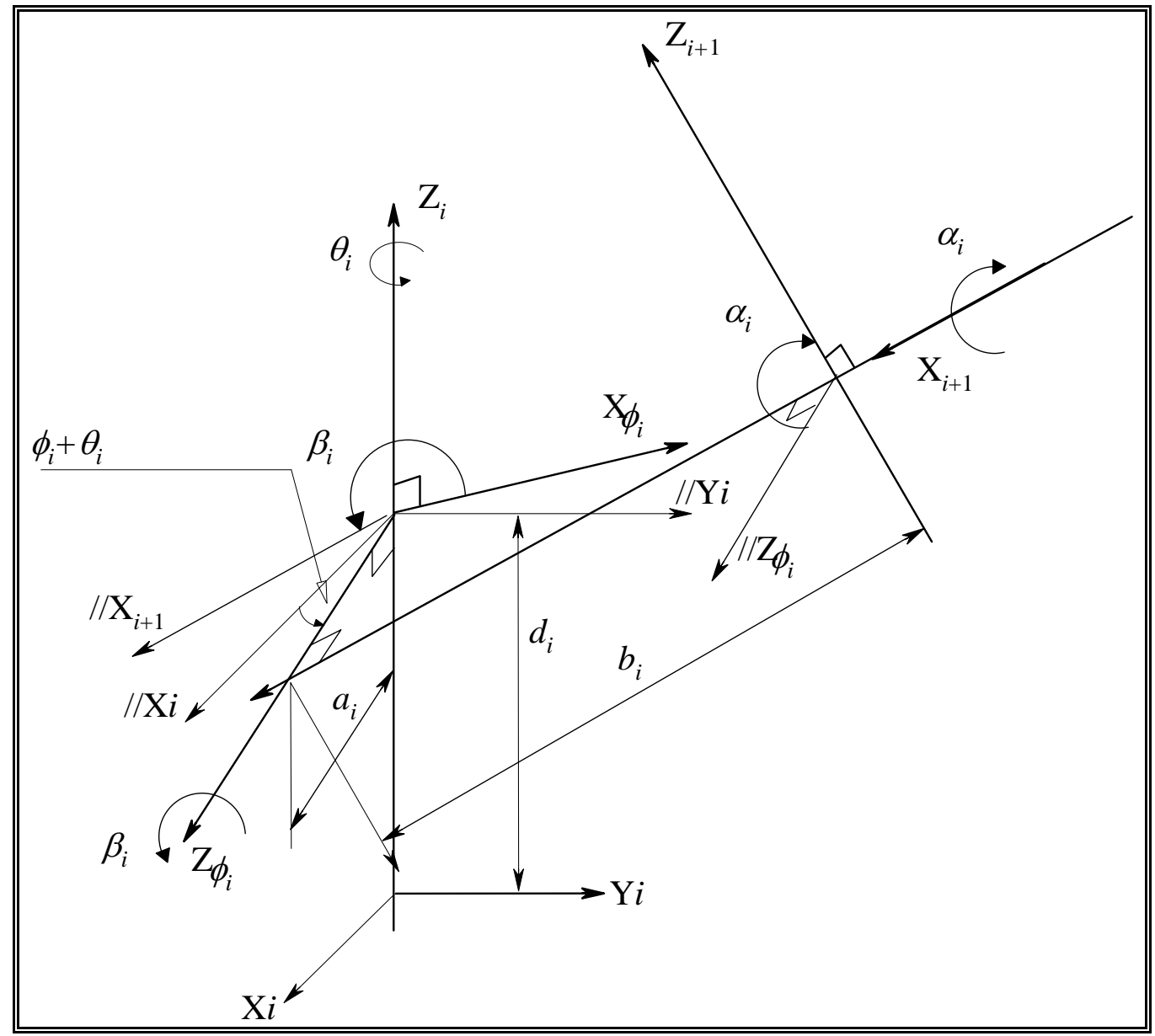

Figure (11): The Kinematic Notation of the $\phi$-Model. 


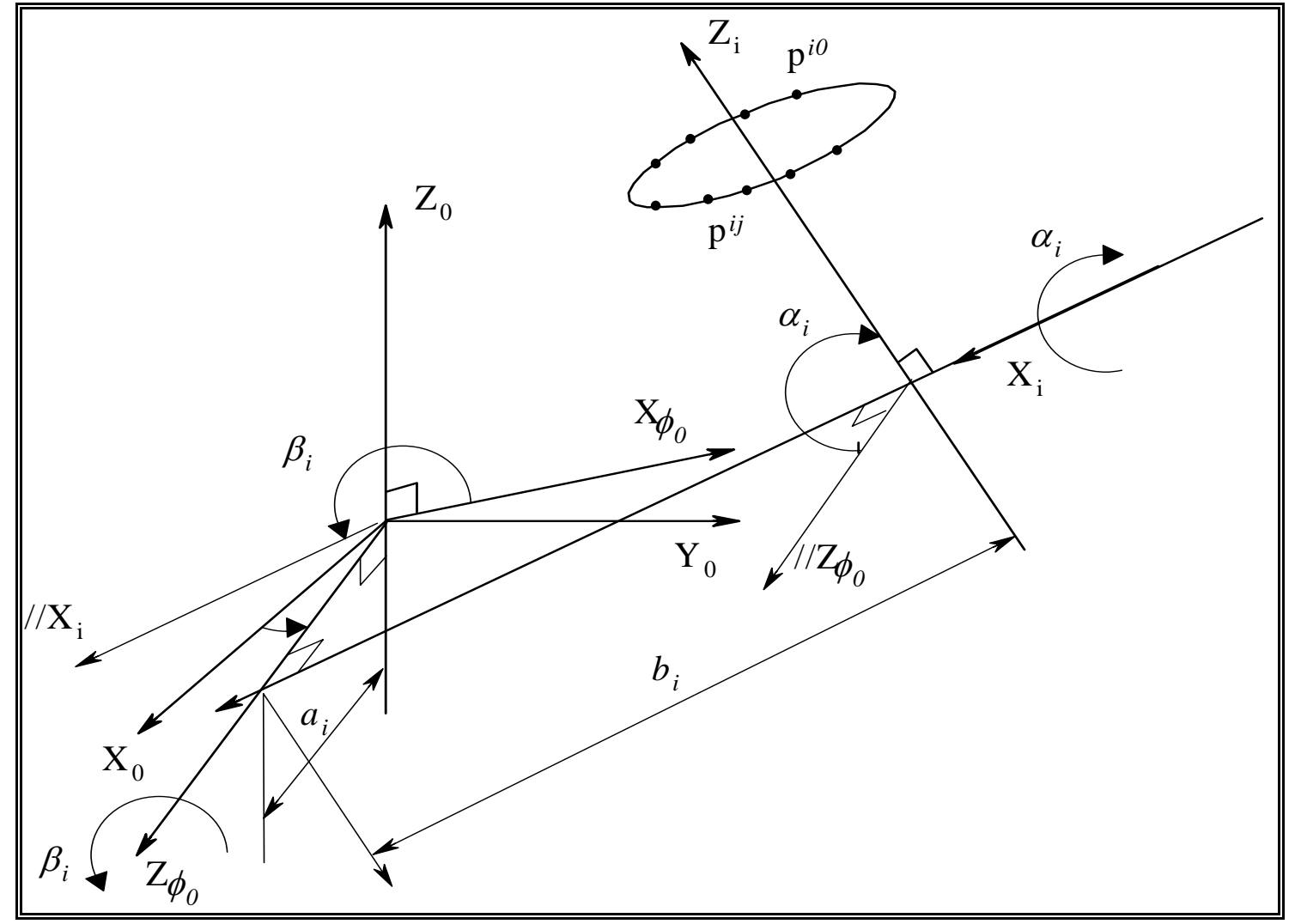

Figure (1): Identifying an Axis of Rotation. 


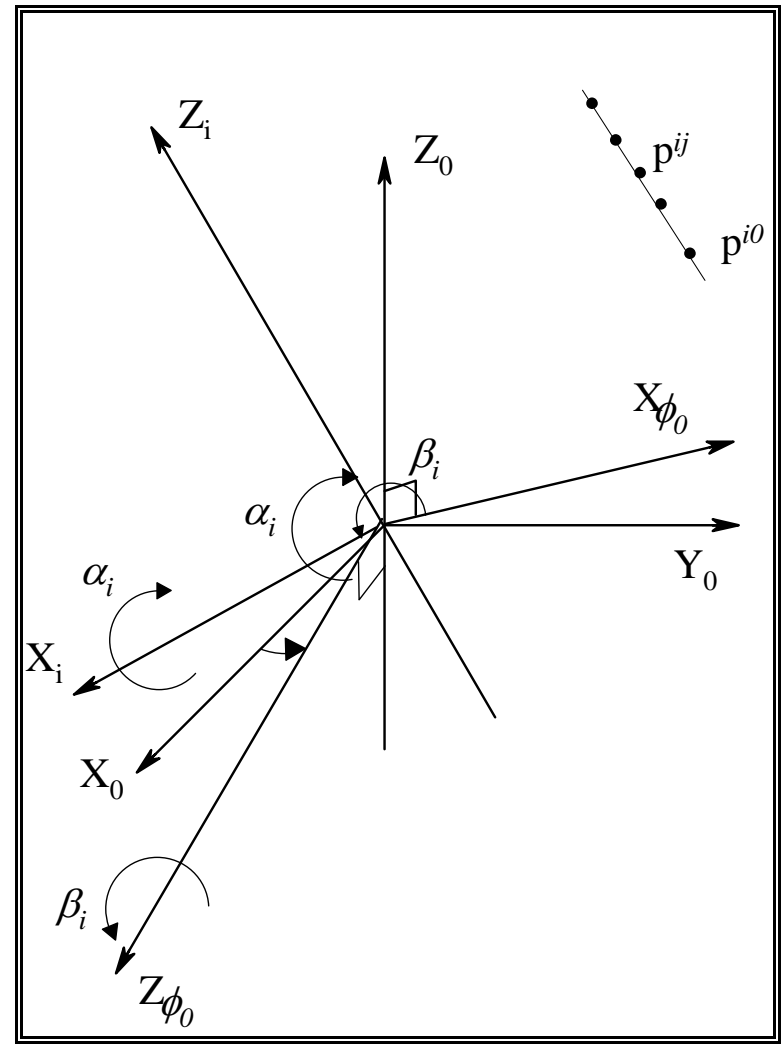

Figure (2): Identifying an Axis of Sliding. 


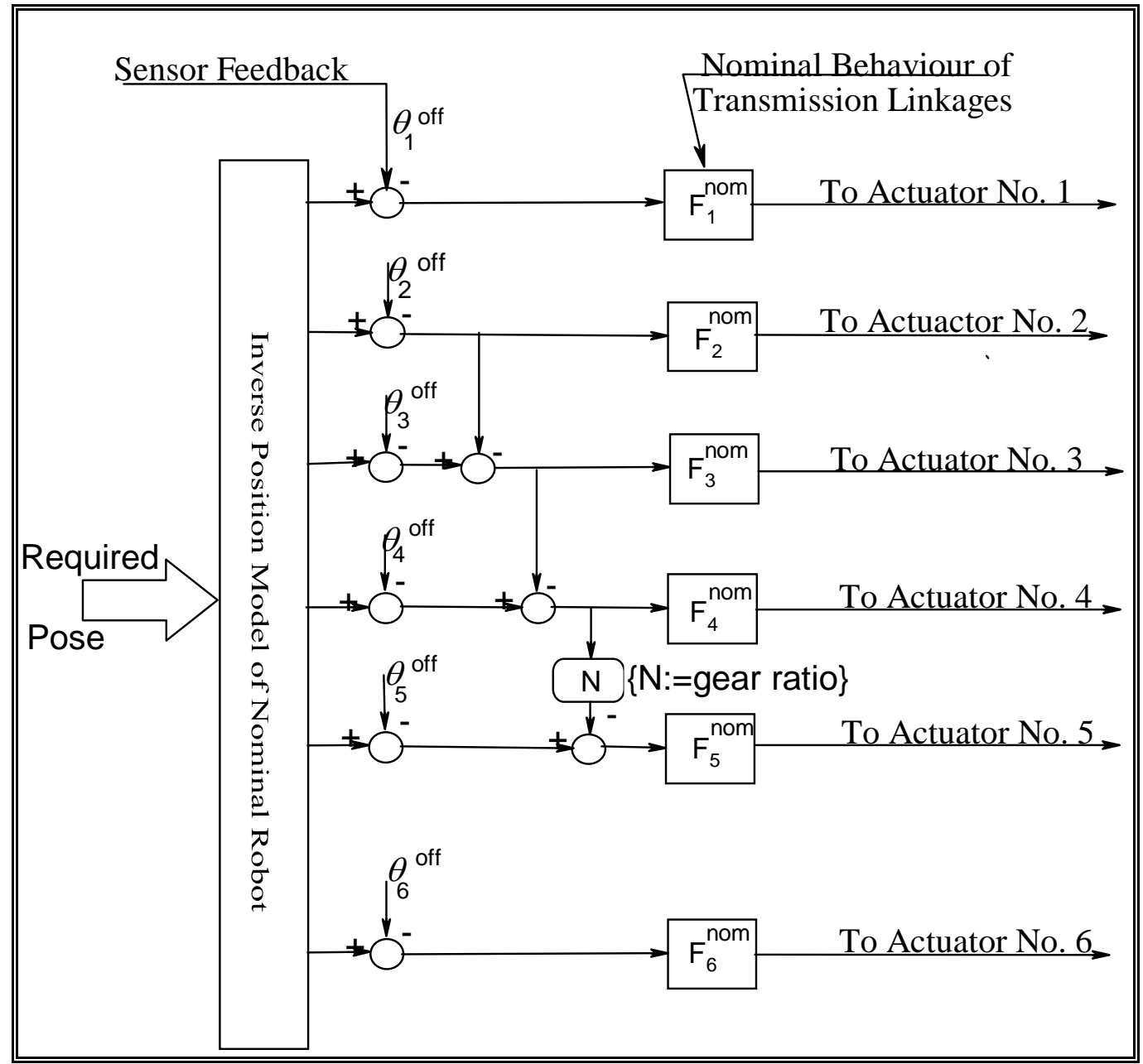

Figure (3): Control and Joint Dependencies in ASEA IRB/L6 Robot. 


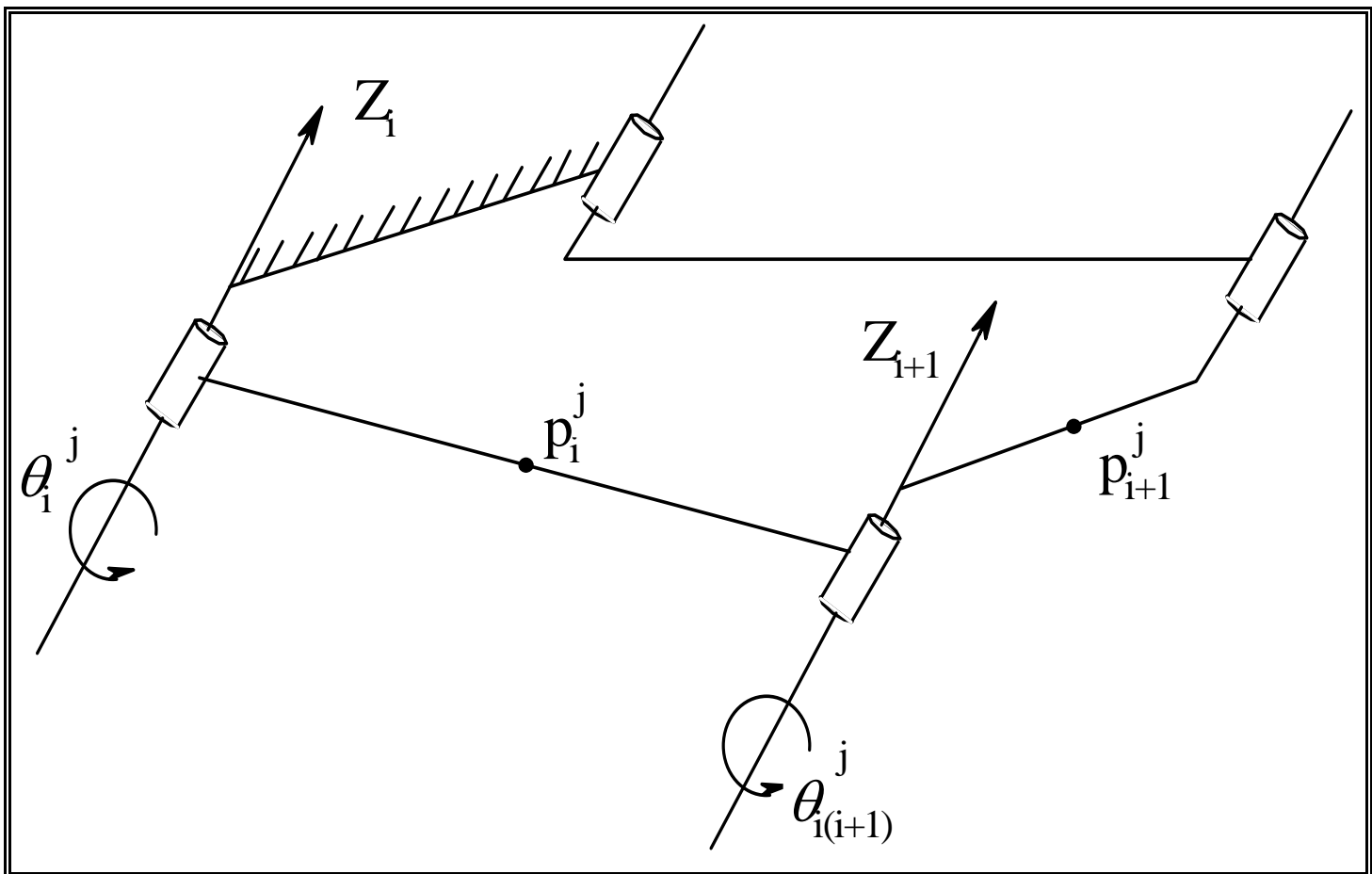

Figure (4): Transmission of Passive Motions in Manipulators. 


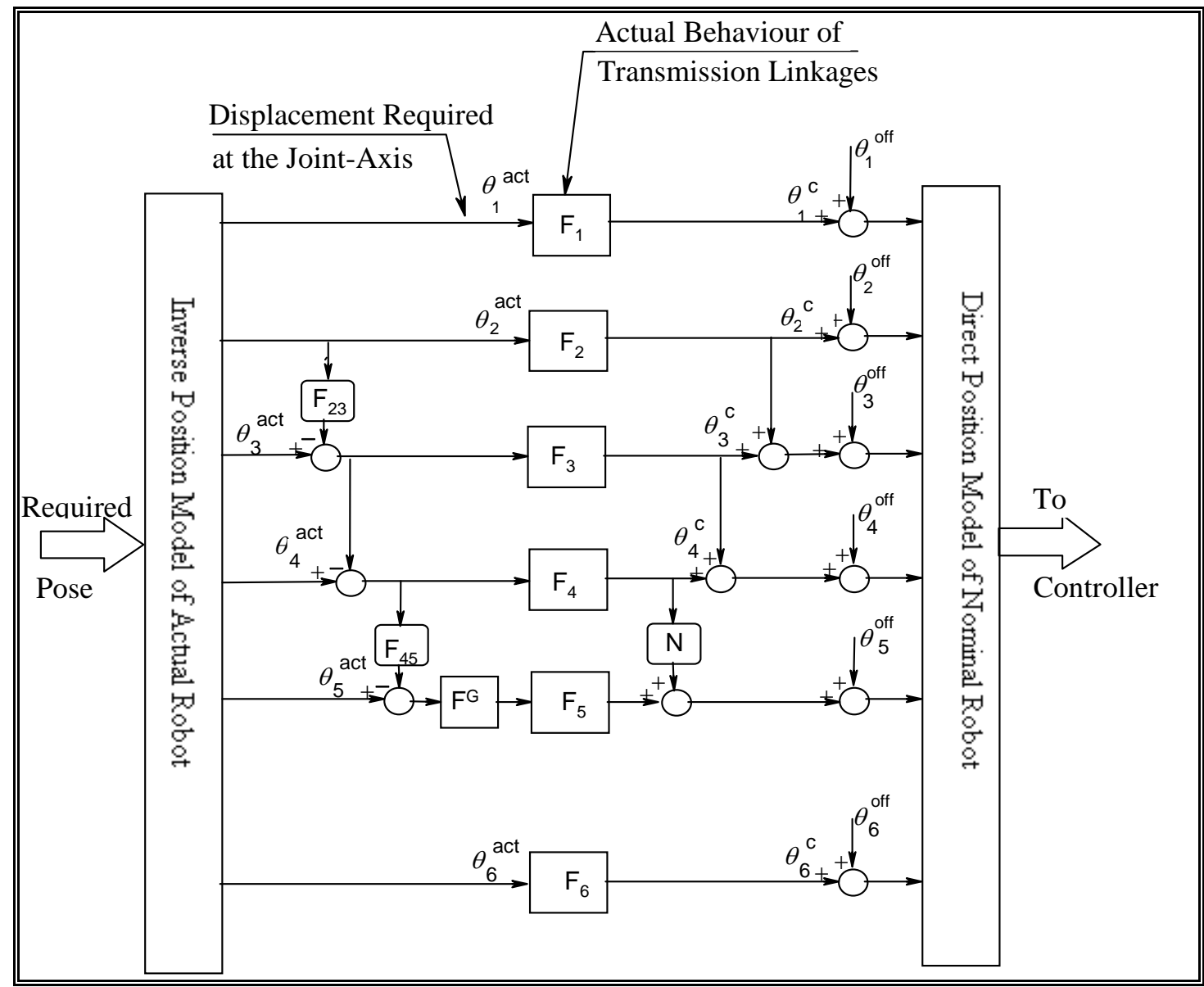

Figure (5): Compensation Strategy Proposed for the Calibrated Robot. 


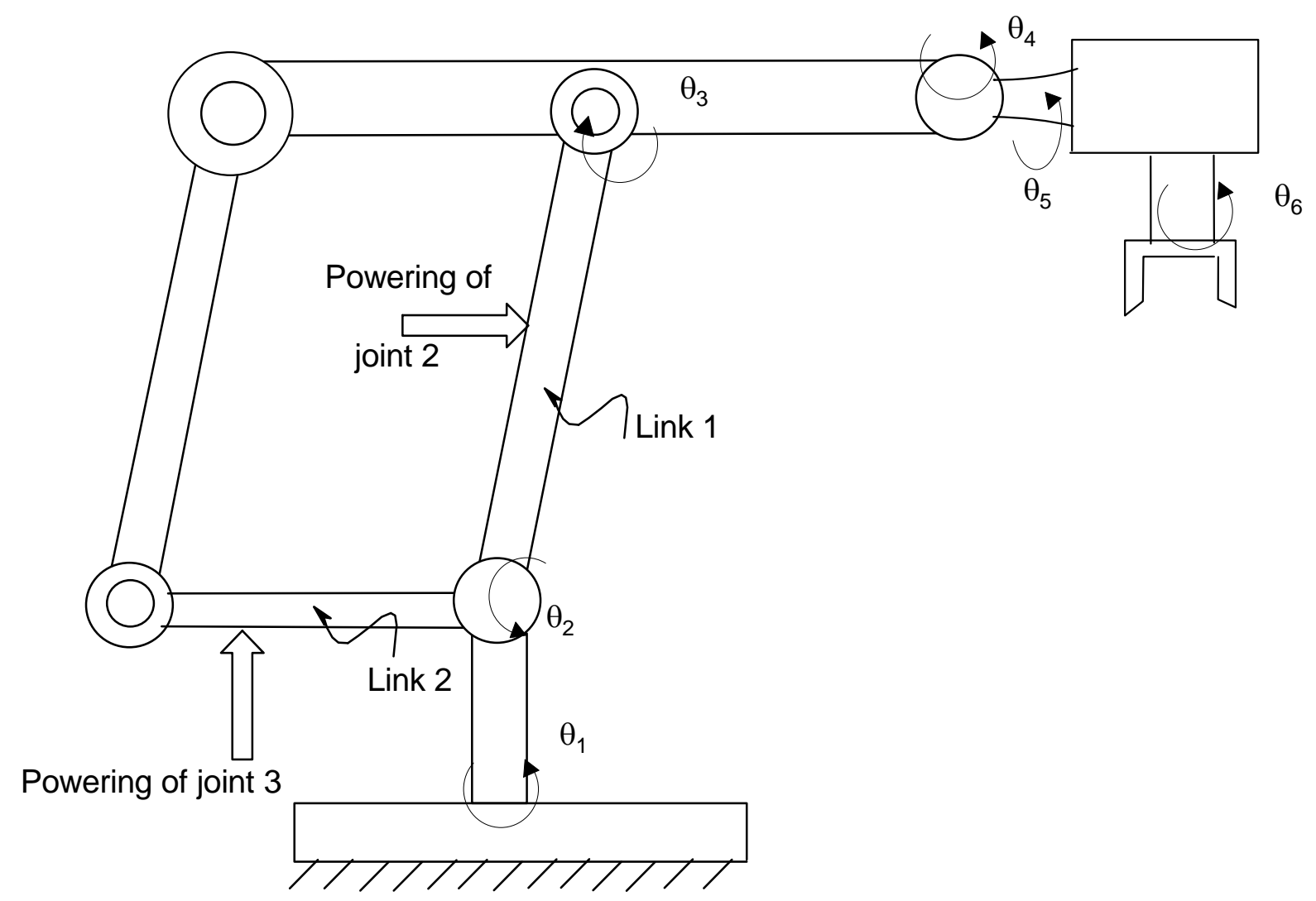

Figure (6): A Schematic Diagram of the Robot Under Study. 


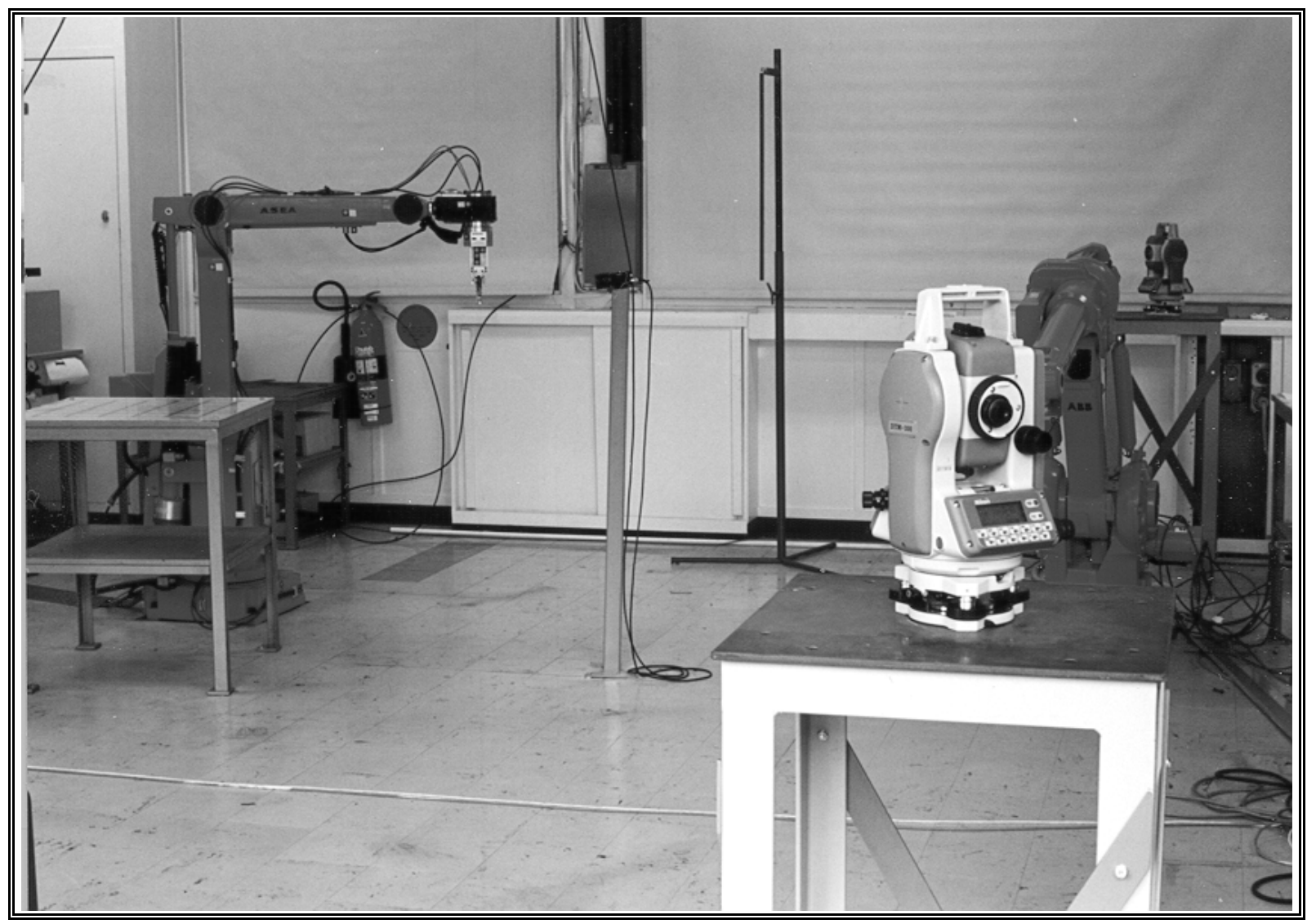

Figure (7): Set-up for Robot Calibration. 


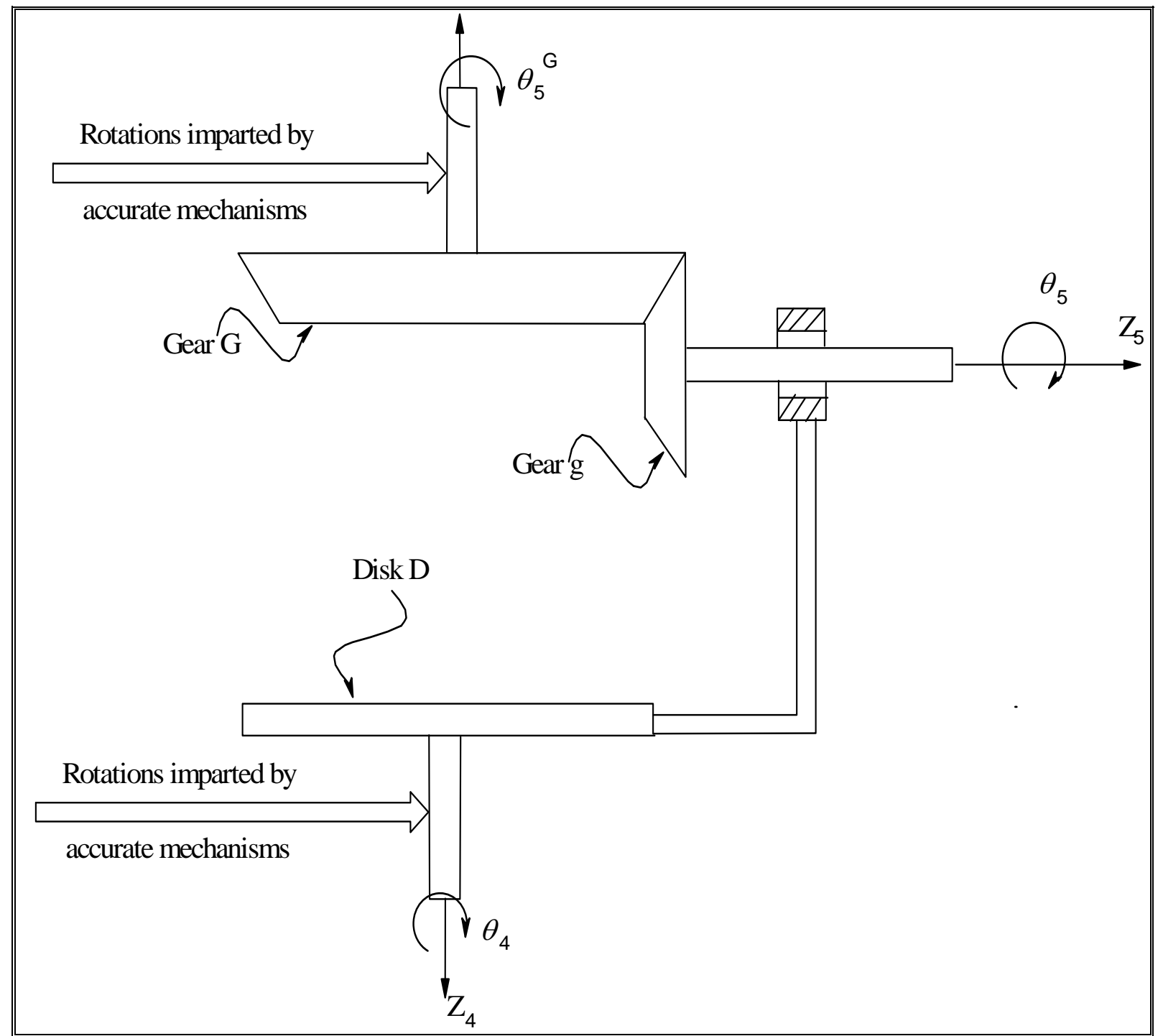

Figure (8): A Schematic Diagram of the Wrist-Mechanism. 


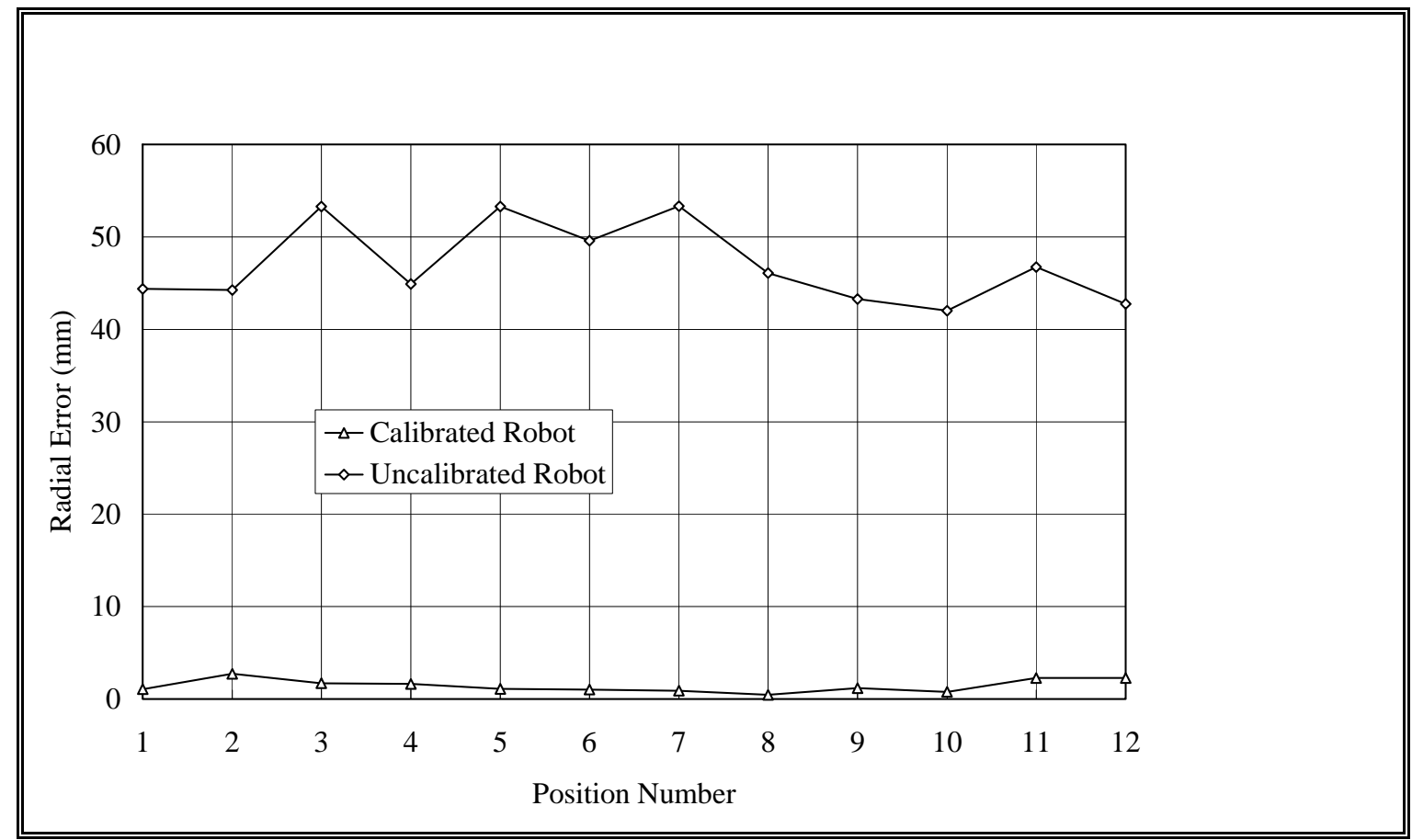

Figure (9): Radial Error of Calibrated and Nominal Robots. 


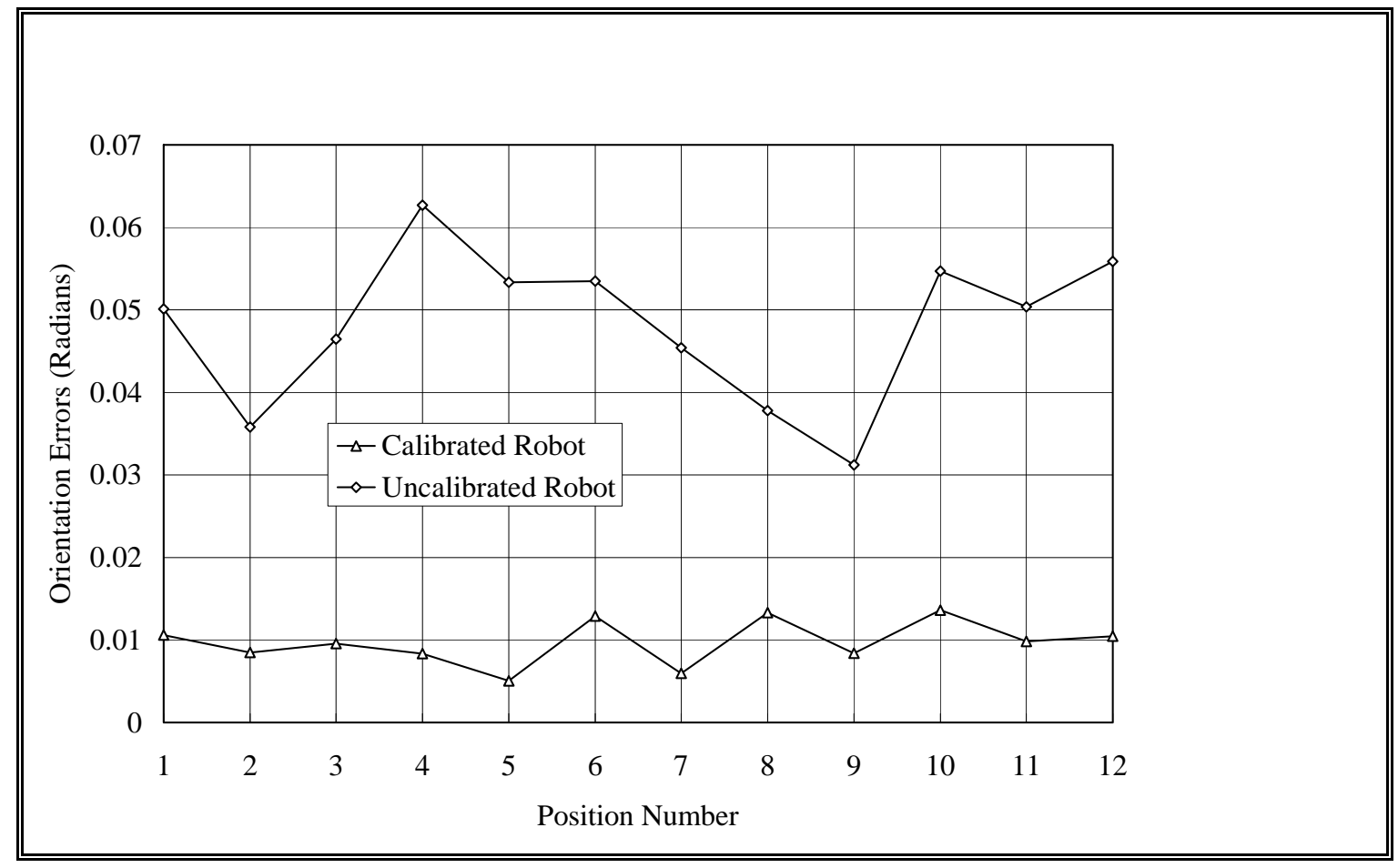

Figure (10): Orientation Error of Calibrated and Nominal Robot. 
Table (1): Aspects of Data Collection.

\begin{tabular}{|l|l|l|l|}
\hline $\begin{array}{l}\text { Number of Joint-Axis } \\
\text { (in order of Calibration) }\end{array}$ & $\begin{array}{l}\text { Displacement Span } \\
\text { Used (deg.) }\end{array}$ & $\begin{array}{l}\text { Number of } \\
\text { Intervals }\end{array}$ & $\begin{array}{l}\text { No of Points } \\
\text { Observed }\end{array}$ \\
\hline 6 & 50 & 16 & $1 \times 17$ \\
5 & 60 & 15 & $1 \times 16$ \\
4 & 90 & 15 & $2 \times 16^{*}$ \\
3 & 45 & 15 & $1 \times 16$ \\
2 & 45 & 15 & $2 \times 16^{*}$ \\
1 & 48 & 15 & $1 \times 16$ \\
\hline
\end{tabular}

* An extra point was observed at each location to calculate the kinematic dependencies.

Table (2): $\phi$-Model Parameters of the ASEA IRB/L6 Robot.

\begin{tabular}{|l|l|l|l|l|l|l|l|l|}
\cline { 2 - 8 } \multicolumn{1}{c|}{} & \multicolumn{4}{c|}{ Nominal Manipulator } & \multicolumn{4}{c|}{ Actual Manipulator } \\
\hline 1 & $a_{i}(\mathrm{~m})$ & $b_{i}(\mathrm{~m})$ & $\alpha_{i}(\mathrm{deg})$ & $\beta_{i}(\mathrm{deg})$ & $a_{i}(\mathrm{~m})$ & $b_{i}(\mathrm{~m})$ & $\alpha_{i}(\mathrm{deg})$ & $\beta_{i}(\mathrm{deg})$ \\
2 & 0.0 & 0.0 & 90.0 & -90.0 & 0.00232 & 0.0 & 90.0 & -90.378 \\
3 & -0.690 & 0.0 & 90.0 & -180.0 & -0.6931 & 0.00487 & 89.828 & -179.54 \\
4 & -0.670 & 0.0 & 90.0 & -180.0 & -0.6727 & 0.00162 & 90.4213 & -179.99 \\
5 & 0.0 & 0.0 & 90.0 & -90.0 & -0.0007 & 0.00297 & 89.8033 & -89.921 \\
\hline
\end{tabular}

